\title{
"SCREAM" THE SOCIAL MEDIA NETWORK
}

\author{
Manav Vasisth \\ BCA Industry Oriented \\ School of Computing Science \\ and Engineering(SCSE) \\ Meerut, India
}

\author{
Aaditya Vijay Srivastava \\ Integrated $\mathrm{BCA}+\mathrm{MCA}$ \\ School of Computing Science \\ and Engineering(SCSE) \\ Varanasi, India
}

\author{
Udit Garg \\ BCA \\ School of Computing Science \\ and Engineering(SCSE) \\ Etawa, India
}

\begin{abstract}
The framework proposed in this paper is a serious answer for the social media as now days all fake and promote all the negativity. Our site is based on the, to help people who are suffering from the mental health issues so in our platform the user can tell their views to the and their issues by they are suffering. This is for stopping the negativity which are now days more growing in todays world.
\end{abstract}

\section{INTRODUCTION}

SCREAM is a next generation social media platform but with a difference. A place where toxicity, hate, cruelty has no place to sustain. We know you want someone to listen, to understand.

A large section of society doesn't acknowledge mental health issue.

In this rapid growing technological era, we are surrounded by social media starting from facebook, twitter, instagram to whatsapp negativity is gradually taking its higher place. SCREAM will play a vital role in development of POSITIVE social networking and Mental health. Consequently. Here we will be using sentiment analysis to recognize the emotions and help in reducing negativity. The machine learning model will never bound user to express their views but cuss words, hate, radicalism will not be tolerated. It will respect every emotions and religion sentiments and ethos. detected information as for the typical or indicated scopes of specific boundaries. The inserted framework is a coordination of sensor gadgets, remote correspondence which empowers the client to distantly get to the different boundaries and store the data in cloud.

Mental health could be a major concern worldwide and India isn't far behind in sharing this. If we evaluate developments within the field of mental state, the pace appears to be slow. Dr. Brock Chisholm, the primary Director-General of the planet Health Organization (WHO), in 1986, had presciently declared that "without mental state there may be no true physical health over 60 years later, the scenario has not altered substantially. About $15 \%$ of the worldwide burden of disease is attributed to neuropsychiatric disorders. The burden of mental disorders is probably going to own been underestimated due to inadequate appreciation of the inter-play between psychopathy and other health disorders.

There remain considerable problems with priority-setting supported the burden of health problems and of addressing inequalities in reference to determinants and solutions for health problems.

Progress in mental health service delivery has been slow in most low- and middle-income countries, the low numbers of those trained in mental health care; and the lack of mental health perspective in public-health leadership.

There have been numerous calls for invoking political will, for enhancing advocacy and for galvanizing community participation; all with scant improvement in outcomes.

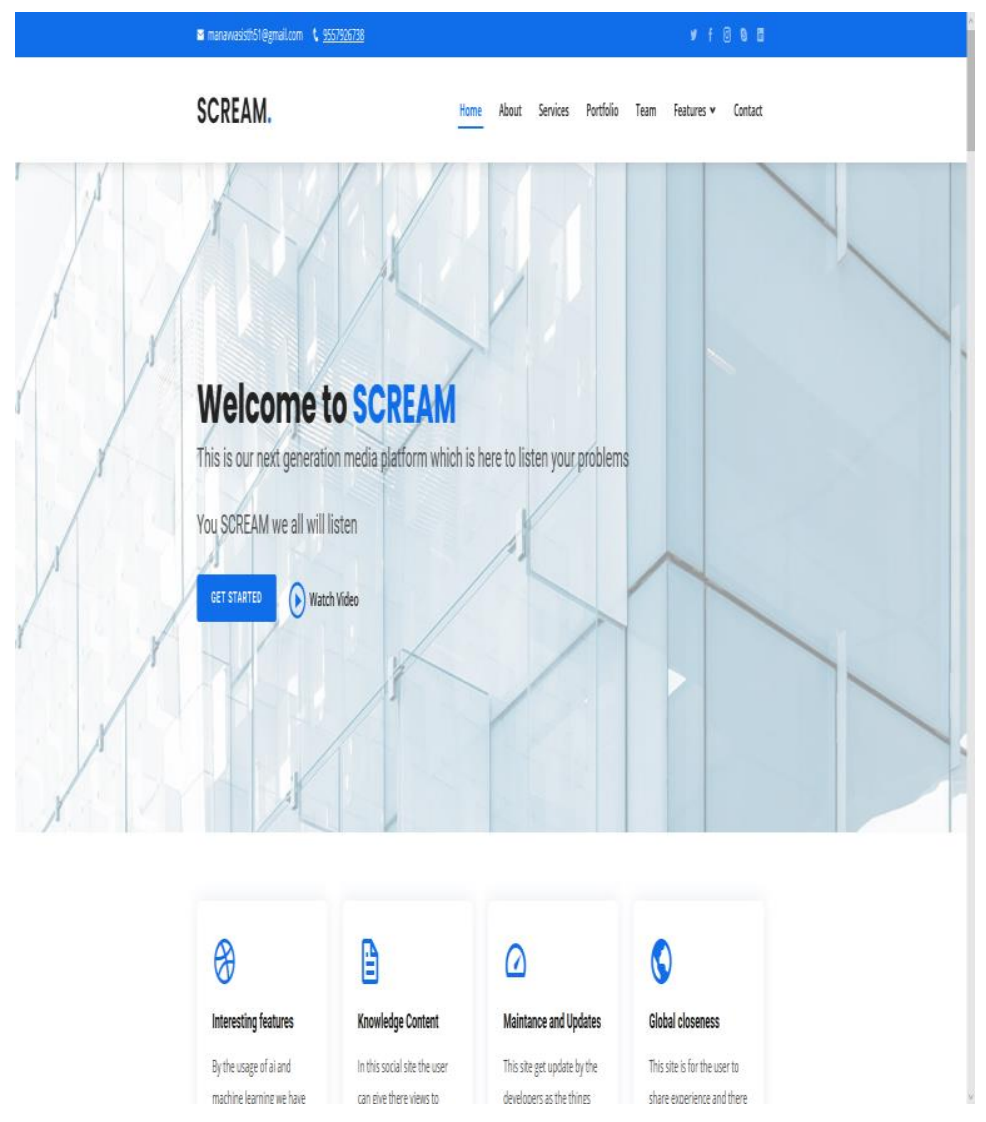

\section{TOOL AND DEVELOPMENT}

MERN STACK: MERN Stack could be a Javascript Stack that's used for easier and faster deployment of full-stack web applications. 


\section{International Journal of Engineering Applied Sciences and Technology, 2021 \\ Vol. 5, Issue 10, ISSN No. 2455-2143, Pages 147-150 \\ Published Online February 2021 in IJEAST (http://www.ijeast.com)}

MERN Stack comprises of 4 technologies namely: MongoDB, Express, React and Node.js, computing is employed in news tell voice assistant to inform the most recent news with the covid 19 updates and natural events it's designed to form the event process smoother and easier.

Each of those 4 powerful technologies provides an end-toend framework for the developers to figure in and every of those technologies play a giant part within the development of web applications.

MongoDB may be a NoSQL database where each record could be a document comprising of key-value pairs that are kind of like JSON (JavaScript Object Notation) objects. MongoDB is flexible and allows its users to make schema, databases, tables, etc. It stores the info of the linked frontends of the users. Once MongoDB is installed, users can make use of Mongo shell moreover.

Mongo shell provides a JavaScript interface through which the users can interact and perform operations to induce the info direct within the database of the developer's page so that they can make changes, Fast to being a documentoriented database, easy to index documents. Scalability of the massive data will be handled by dividing it into several machines.

Use of JavaScript in Mongo DB uses JavaScript which is that the biggest advantage.

Flexible Document Model in Mongo DB supports document-model which is quicker and easier.

Express may be a Node.js framework. instead of writing the code using Node.js and creating many Node modules, Express makes it simpler and easier to put in writing the back-end code. Express helps in designing great web applications and APIs. To link the info at the backend of the websites and express supports many middle wares which makes the code shorter and easier to jot down.

\section{Robust API}

Create a brand new folder to begin your express project and kind below command within the electronic communication to initialize a package. json file. Accept the default settings and continue. To link new API address to link maps and differing kinds to indicate multiple things within the website to form interesting and attractive

\section{React: Front-End Library}

React could be a JavaScript library that's used for building user interfaces. React is employed for the event of singlepage applications and mobile applications due to its ability to handle rapidly changing data. React allows users to code in JavasScript and build UI components.

Virtual DOM - A virtual DOM object could be a representation of a DOM object. Virtual DOM is really a duplicate of the first DOM. Any modification within the web application causes the complete UI to re-render the virtual DOM. Then the difference between the initial DOM and this virtual DOM is compared and therefore the changes are made accordingly to the first DOM.
JSX - Stands for JavaScript XML. it's an HTML/XML JavaScript Extension which is employed in React. Makes it easier and simpler to put in writing React components.

Components - React JS supports Components. Components are the building blocks of UI wherein each component encompasses a logic and contributes to the general UI. These components also promote code reusability and make the general web application easier to grasp.

High Performance - Features like Virtual DOM, JSX and Components makes it much faster than the remainder of the frameworks out there.

Developing Android/Ios Apps - With React Native you'll be able to easily code Android-based or IOS-Based apps with just the knowledge of JavaScript and ReactJS.

You can start your react application by first installing "create-react-app" using npm or yarn.

Node.js: JS Runtime Environment

Node.js provides a JavaScript Environment which allows the user to run their code on the server (outside the browser). Node pack manager i.e. npm allows the user to settle on from thousands of free packages (node modules) to download.

Open source JavaScript Runtime Environemnt Single threading - Follows one threaded model.

Data Streaming

Fast - Built on Google Chrome's JavaScript Engine, Node.js incorporates a fast code execution.

\section{Highly Scalable}

Initialize a Node.js application by typing runing the below command within the command window. Accept the quality settings. (Jasraj, 2020)

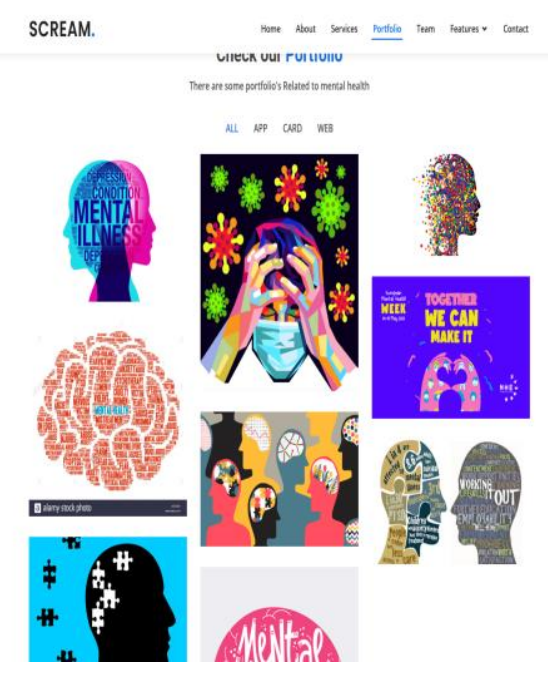




\section{International Journal of Engineering Applied Sciences and Technology, 2021 Vol. 5, Issue 10, ISSN No. 2455-2143, Pages 147-150 \\ Published Online February 2021 in IJEAST (http://www.ijeast.com)}

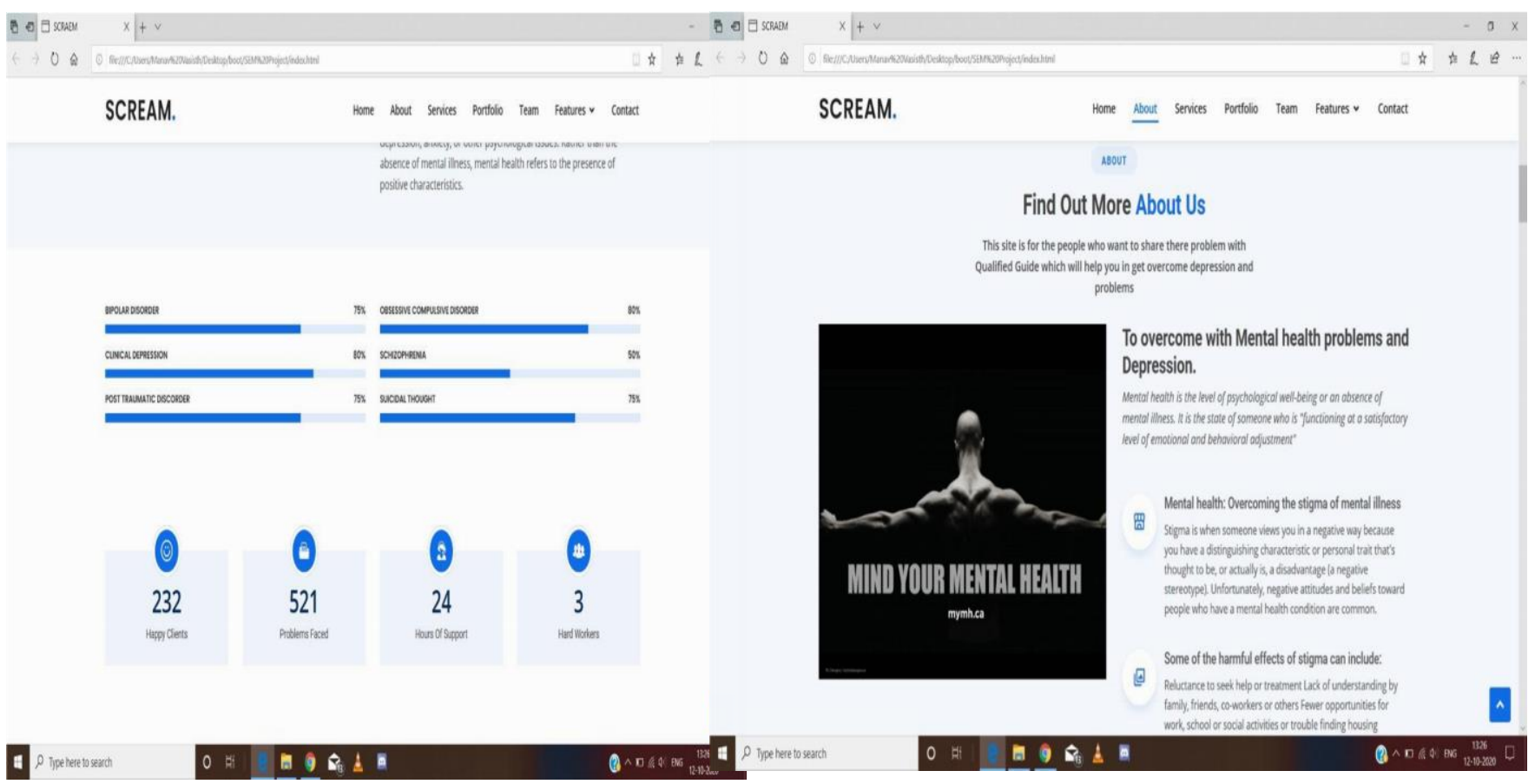

\section{NATURAL LANGUAGE PROCESS}

Natural language processing may be a subfield of linguistics, engineering, and computing concerned with the interactions between computers and human language, particularly a way to program computers to process and analyzelarge amounts of language data

There's lots of tongue data out there in various forms and it might get very easy if computers can understand and process that data. we are able to train the models in accordance with expected output in several ways. Humans are writing. For thousands of years, there are lots of literature pieces available, and it might be great if we make computers understand that. But the task isn't visiting be easy. There are various challenges floating out there like understanding the proper meaning of the sentence, correct

Computers can't truly understand the human language. If we feed enough data and train a model properly, it can distinguish and take a look at categorizing various parts of speech (noun, verb, adjective, supporter, etc...) supported previously fed data and experiences. If it encounters a brand new word it tried making the closest guess which might be embarrassingly wrong few times.

\section{CONCLUSION}

As highlighted during this commentary, social media has become a very important a part of the lives of the many individuals living with mental disorders. Many of those individuals use social media to share their lived experiences with mental state, to hunt support from others, and to go looking for information about treatment recommendations, accessing mental state services and addressing symptoms ,As the field of digital psychological state advances, the wide reach, simple access, and recognit ion of social media platforms might be accustomed allow individuals in need of mental state services or facing challenges of mental state to access evidence-based treatment and support. With these quite websites help the users to grasp the problems and also the disorders which they're addressing. At these platform the users have a special variety of image from there world so that they can show the personalities of themselves. And it help the people to be in there comfort space also

\section{FUTURE SCOPE}

This will reason that the constant information effectively supportive towards the society that what number people are there and these reasonably platform will help the opposite one who recovered from these issues they will guide the opposite person also of utilizations like Data checking, sending and controlling of knowledge at the positioning.

\section{REFERENCES}

1. Abdel-Baki, A., Lal, S., Charron, D.-C., Stip, E., \& Kara, N. (2017). Understanding access and use of technology among youth with first-episode psychosis to inform the development of technology-enabled therapeutic interventions. Early Intervention in Psychiatry, 11(1), 72-76. 


\section{International Journal of Engineering Applied Sciences and Technology, 2021 \\ Vol. 5, Issue 10, ISSN No. 2455-2143, Pages 147-150 \\ Published Online February 2021 in IJEAST (http://www.ijeast.com)}

2. Ahmed, Y. A., Ahmad, M. N., Ahmad, N., \& Zakaria, N. H. (2019). Social media for knowledge-sharing: a systematic literature review. Telematics and Informatics, 37, 72-112.

Alhajji, M., Bass, S., \& Dai, T. (2019). Cyberbullying, mental health, and violence in adolescents and associations with sex and race: data from the 2015 youth risk behavior survey. Global Pediatric Health, 6, 2333794X19868887.

3. Alvarez-Jimenez, M., Bendall, S., Lederman, R., Wadley, G., Chinnery, G., Vargas, S., Larkin, M., Killackey, E., McGorry, P., \& Gleeson, J. F. (2013). On the HORYZON: moderated online social therapy for long-term recovery in first episode psychosis. Schizophrenia Research, 143(1), 143-149.

4. Alvarez-Jimenez, M., Gleeson, J., Bendall, S., Penn, D., Yung, A., Ryan, R., et al. (2018). Enhancing social functioning in young people at ultra high risk (UHR) for psychosis: a pilot study of a novel strengths and mindfulnessbased online social therapy. Schizophrenia Research, 202, 369-377.

5.Alvarez-Jimenez, M., Bendall, S., Koval, P., Rice, S., Cagliarini, D., Valentine, L., et al. (2019). HORYZONS trial: protocol for a randomised controlled trial of a moderated online social therapy to maintain treatment effects from first-episode psychosis services. BMJ Open,9(2), e024104..

6.Abramowitz JS. Treatment of obsessive-compulsive disorder in patients who have comorbid major depression. Journal of Clinical Psychology. 2004;60:11331141. [PubMed]

7.Abramowitz JS, Schwartz SA, Moore KM, et al. Obsessive-compulsive symptoms in pregnancy and the puerperium: a review of the literature. Journal of Anxiety Disorders. 2003;17:461-478. [PubMed]

8.Adler R, Vasiliadis A, Bickell N. The relationship between continuity and patient satisfaction: a systematic review. Family Practice. 2010;27:171-178. [PubMed]

9.Adli M, Bauer M, Rush AJ. Algorithms and collaborativecare systems for depression: are they effective and why? A systematic review. Biological Psychiatry. 2006;59:10291038. [PubMed]

10.Aertgeerts B, Buntinx F, Kester A. The value of the CAGE in screening for alcohol abuse and alcohol dependence in general clinical populations: a diagnostic meta-analysis. Journal Clinical Epidemiology. 2004;57:3039. [PubMed]

11.Agarwal G, Crooks VA. The nature of informational continuity of care in general practice. British Journal of General Practice. 2008;58:e17-e24. [PMC free article] [PubMed]
12.AGREE Collaboration. Development and validation of an international appraisal instrument for assessing the quality of clinical practice guidelines: the AGREE project. Quality and Safety in Health Care. 2003;12:1823. [PMC free article] [PubMed]

13.Akesson KM, Saveman BI, Nilsson G. Health care consumers' experiences of information communication technology: a summary of literature. International Journal of Medical Informatics. 2007;76:633-645. [PubMed]

14.Akiskal HS. A developmental perspective on recurrent mood disorders: a review of studies in man. Psychopharmacology Bulletin. 1986;22:579586. [PubMed]

15.Jasraj(2020) geeksforgeeks for mern stack and api of the project tools for the web designing of the website. 